\title{
Comparative Evaluation of Fractal Dimensions of CBCT Images Obtained with i-CAT Next Generation and PreXion 3D Elite Scanners
}

\author{
Carlos E. P. Toledoํㅜ, Marcio J. S. Campos ${ }^{2}$, Roberto S. M. F. de Oliveira ${ }^{2}$, Robert W. F. Vitral ${ }^{2}$ \\ ${ }^{1}$ Juiz de Fora Federal University, Juiz de Fora, Brazil; ${ }^{2}$ Department of Orthodontics, Juiz de Fora Federal University, \\ Juiz de Fora, Brazil
}

Correspondence to: Carlos E. P. Toledo, carlostoledo1@hotmail.com

Keywords: Bone Density, X-Ray Computed Tomography, Fractals

Received: November 27, $2018 \quad$ Accepted: December 25, $2018 \quad$ Published: December 28, 2018

Copyright $\odot 2018$ by authors and Scientific Research Publishing Inc.

This work is licensed under the Creative Commons Attribution International License (CC BY 4.0).

http://creativecommons.org/licenses/by/4.0/

\section{(c) () Open Access}

\section{ABSTRACT}

Comparing Cone-Beam Computed Tomography (CBCT) exams with different fields of view (FOV) are relevant to understand the impact of the inherent image quality on the reliability and accuracy of the diagnostic outcome. This study aimed to comparatively evaluate the results of the fractal analysis of images of the same structure taken in two cone beam computerized scanners with different FOVs. The initial selection of the images used, from two CBCT of the same individual jaw performed in two different scanners, was conducted in the Radiant program (RadiAnt DICOM Viewer) and exported to DICOM format. The selected images were processed in the ImageJ software (US National Institutes of Health), correcting the rotation of the images before conducting fractal analysis. In order to verify the normality of the data, the Shapiro-Wilk test was applied. The Mann-Whitney U, Levene and T tests were applied in order to compare the fractal analysis obtained by the two CBCT scanners. The intraclass correlation coefficient result was 0.988 . The mean of the samples in the three comparisons (mentum samples, mental foramen samples and all included mentum and mental foramen samples) was higher for the PreXion 3D scanner. The $p$ value of the tests was $\mathbf{0 . 0 0 0}$, for the three comparisons, at a $5 \%$ confidence level. The comparison of the fractal dimension averages of the images obtained with i-CAT and PreXion 3D scanners showed statistically significant differences, and higher averages were observed in the fractal analysis of images from PreXion 3D scanner. These results point to the need for a careful analysis of the parameters involved in the acquisition of images when results of fractal analysis resulting from different equipment are compared. 


\section{INTRODUCTION}

In the analysis of craniofacial skeletal structures, conventional radiographic examinations poorly display anatomical features due to the overlapping of bone structures [1]. In these situations, computed tomography scan is the exam of choice, because it eliminates overlapping images and allows cross-sectional visualization. One of the main advantages of cone-beam computed tomography (CBCT) is the reduced radiation dose, which is $40 \%$ lower than in fan beam computed tomography [2]. Furthermore, its clinical application in the field of craniofacial radiology is widely diffused [3, 4].

Tomographic images allow accurate linear and angular (quantitative) measurements of the structures evaluated [5] and also qualitative analysis of bone structures, through alveolar bone mineral density [6-8] and fractal dimension assessment $[9,10]$.

The unit of measurement used in fan beam computed tomography for bone mineral density is the Hounsfield Unit (HU). Each tissue is represented by the amount of X-ray photons absorbed [9]. In CBCT, a non-uniform angular distribution of the intensity of the X-ray beams leads to non-uniformity of $\mathrm{HU}$ measurements. Because the HU values are not applicable in CBCT [11], the fractal analysis has been suggested as an alternative for bone quality assessment [9].

Fractal dimension means strictly the fractional or irrational number that characterizes the geometry of a fractal [12]. Using a fractal model, it is possible to characterize the irregular trabecular structure of the bone tissue through the fractal geometric analysis of its image. Many studies are being conducted using fractal analysis, and their usefulness in dental research has been demonstrated [13].

In clinical practice, the quality of CBCT images and the ability of CBCT to display anatomic features are influenced by a number of variables, such as the scanning unit, the field of view (FOV), examined object, examination time, tube voltage and amperage, and also spatial resolution defined by the voxel size [14]. Librizzi et al., in 2011 [15], found that the diagnostic efficacy of CBCT scans for the evaluation of erosive changes in the temporomandibular joint is highest for 6-inch FOV and lowest for 12-inch FOV. It is important to ponder that the comparison of CBCT examinations with different voxel settings is relevant to understand the impact of the inherent image quality on the reliability and accuracy of the diagnostic outcome.

The present study aims to compare the results of fractal analysis of images of the same structure, performed by two conical beam computerized tomography scanning machines operated with different FOVs.

\section{METHODOLOGY}

\subsection{Material}

The sample consisted of two CBCT scans of the same individual's mandible, performed with i-CAT Next Generation (Imaging Sciences International, Hatfield, PA, USA) and PreXion 3D Elite (PreXion, Inc, San Mateo, CA, USA), with a 1-week interval between the scans. The sample belongs to the Faculty of Dentistry of the Federal University of Juiz de Fora (UFJF) archive. The UFJF Research Ethics Committee, under the consubstantiated statement number 1.998.591, approved this study.

\subsection{Method}

Imaging was performed with i-CAT Next Generation and PreXion 3D Elite.

i-CAT Next Generation settings were: field of view (FOV) $130 \times 160 \mathrm{~mm}$; tube voltage $120 \mathrm{kVp}$; tube current 3 - $7 \mathrm{~mA}$; voxel $0.25 \mathrm{~mm}$; rotation time $26.9 \mathrm{~s}$. PreXion 3D Elite settings were: field of view (FOV) $81 \times 75 \mathrm{~mm}$; tube voltage $90 \mathrm{kVp}$; tube current $4 \mathrm{~mA}$; voxel $0.147 \mathrm{~mm}$; rotation time $19 \mathrm{~s}$. While undergoing $\mathrm{CBCT}$, the individual was seating, with his chin on the appropriate rest, Frankfort plane parallel to the ground surface, sagittal plane perpendicular to the ground surface, and mandible in the maximum intercuspal position. The field of view was positioned so that the mandible body occupied its mid vertical third. The images provided by the two CBCT scanners were stored in multiple uncompressed DICOM format. 


\subsubsection{Selection and Determination of Images}

The initial selection of the images was performed in the Radiant program (RadiAnt DICOM Viewer, version 3.4.1). All images used were from the mandible in the axial plane, from the first inferior image that contained the mentum, passing through the image of the left mental foramen, to the last upper image containing the image of the right mental foramen completely delimited by cortical bone. After the selection of the images, they were exported to DICOM format.

\subsubsection{Image Rotation Correction}

When comparing the images from the two CBCT scanners in the Image J program (US National Institutes of Health, Bethesda, MD, version 1.51a), it was noticed that there were small positioning discrepancies between the devices, therefore causing small rotations in the three cutting planes. Thus, using element 41 as a reference, the rotation of the mesio-distal incisal axis was initially corrected to obtain its horizontal position in the image, using the rotation tool. In the new sets of images created, new slices from left to right were made in order to obtain new images, which were orthogonal to the mesio-distal axis of element 41. In these sliced-images, the shape of the root canal was evaluated and all images were rotated so that the long axis of the root canal of element 41 was vertical. In these two new sets of images, new slices were made from left to right, and in the two frontal images from the two CBCT scanners which showed the long axis of the root canal of element 41, the vertical inclination of its long axis was evaluated and all images were rotated so that the long axis of the root canal of the element 41 was completely vertical. Finally, in these two new sets of images, new top-down slices were made, obtaining then two sets of images which were orthogonal to the root canal of element 41, in which all the fractal analysis were performed.

\subsubsection{Comparative Evaluation Using Fractal Analysis}

The two sets of images, orthogonal to the root canal of element 41, from both CBCT scanners, were analyzed in the ImageJ program for the determination of the fractal dimensions. Two regions of interest (ROI) were chosen: one anterior in the region of the mentum measuring $10 \times 3.75 \mathrm{~mm}$ and another in the region of the mental foramen $\mathrm{D}$ measuring $4 \times 4 \mathrm{~mm}$. The coordinates $66.25(\mathrm{x})$ and $27.75(\mathrm{y})$, and 35.01 (x) and 14.29 (y) were used for the selection of the ROI's of the mentum, and the coordinates 49.25 (x) and $37.25(\mathrm{y})$, and $15.70(\mathrm{x})$ and 24.18 (y) for the selection of the mental foramen ROI's in the images from i-Cat and PreXion 3D scanners, respectively. This way, we determined that the ROI from the i-Cat images are the same as those from the PreXion 3D images (Figures 1-8). Figures 1-8 represent the first and last images of each region studied for each tomograph.

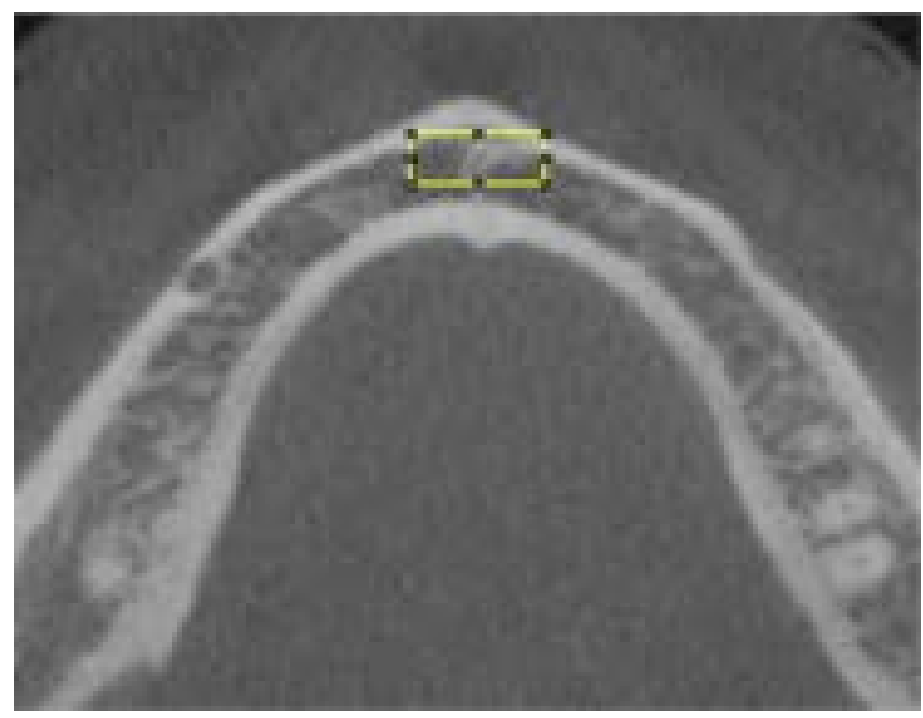

Figure 1. ROI of mentum in i-Cat. 


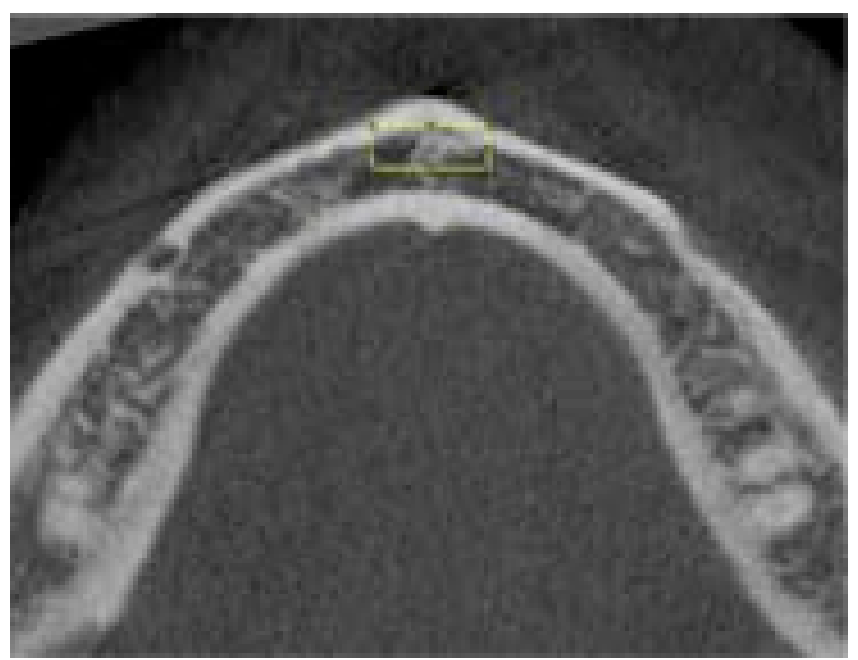

Figure 2. ROI of mentum in PreXion 3D.

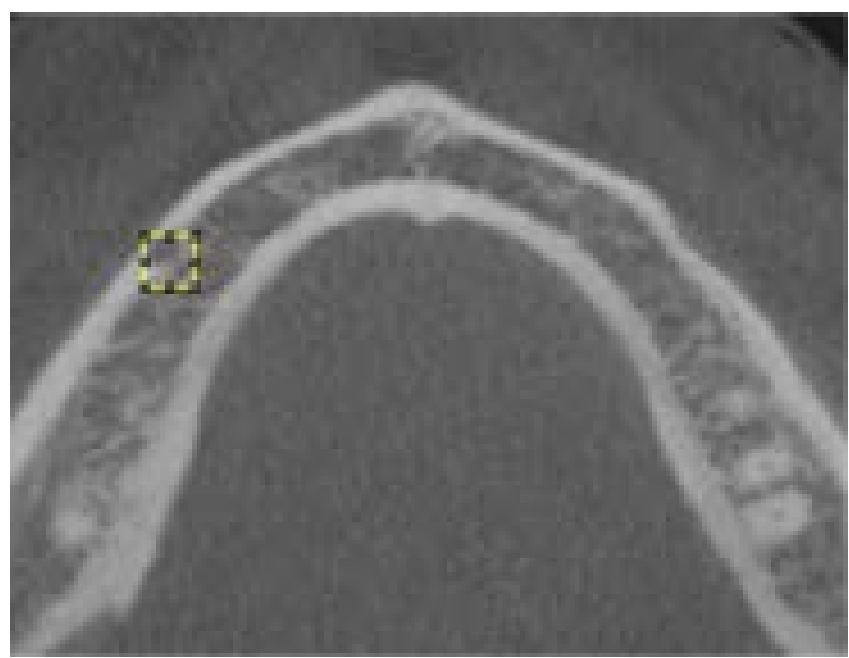

Figure 3. ROI of mental foramen in i-Cat.

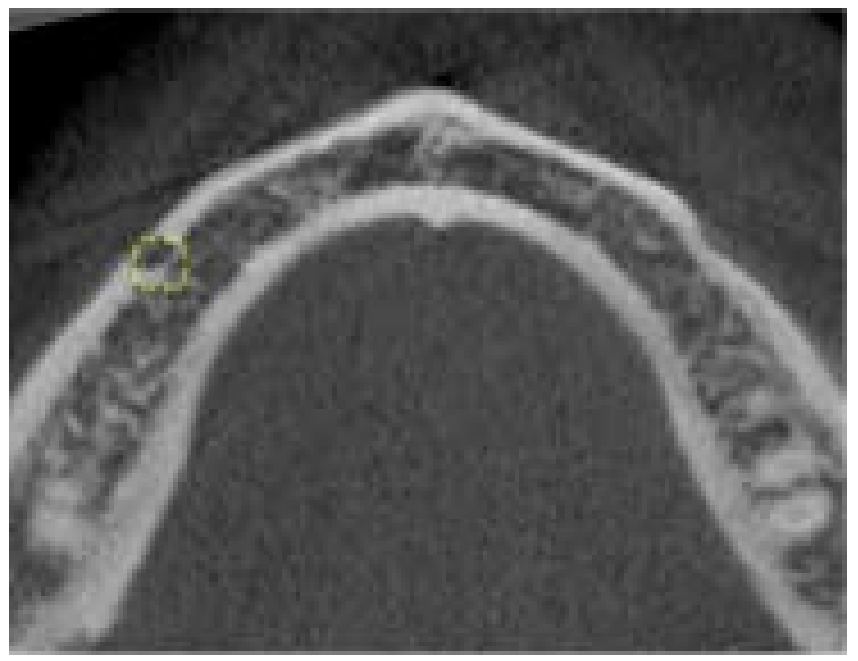

Figure 4. ROI of mental foramen in PreXion 3D. 


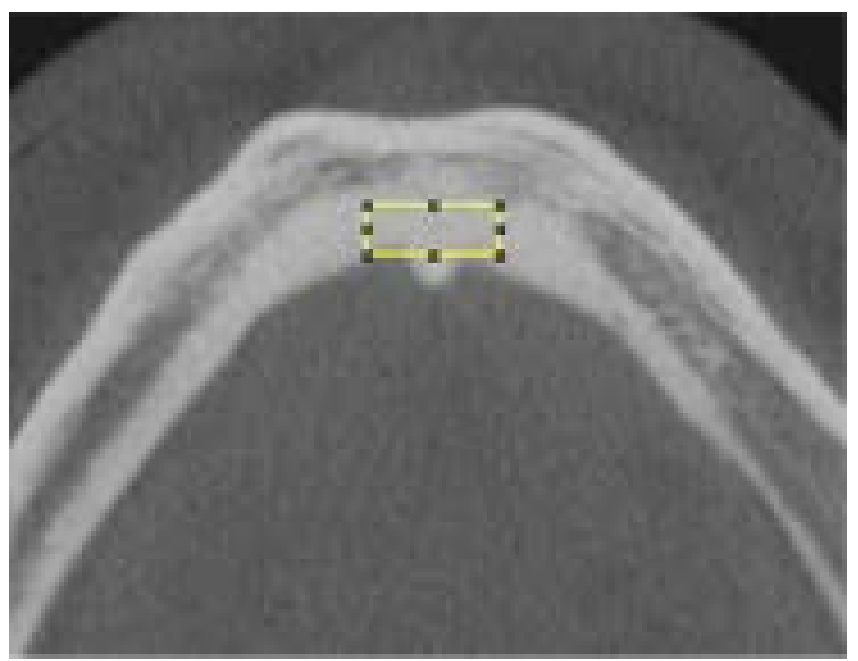

Figure 5. ROI of mentum in the i-Cat.

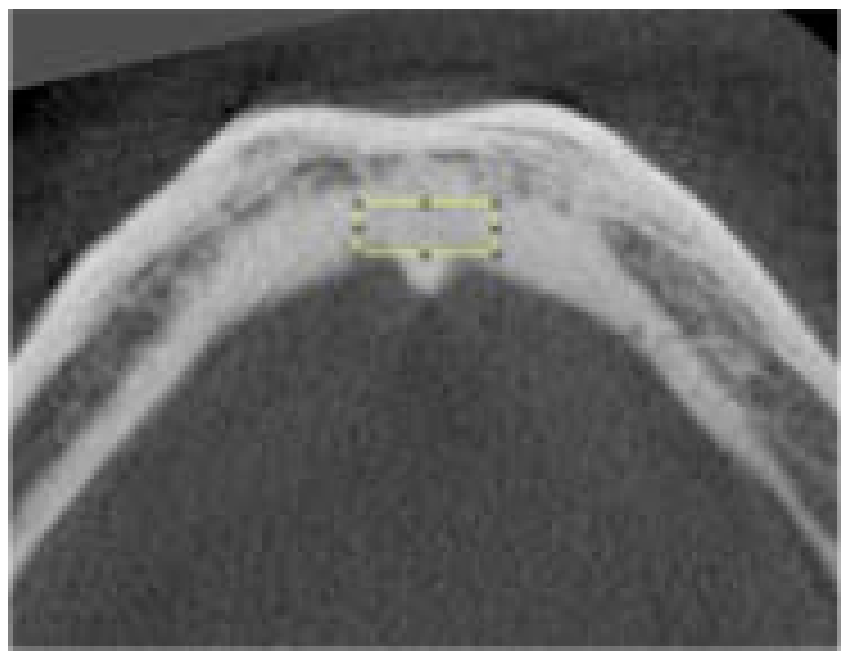

Figure 6. ROI of mentum in PreXion 3D.

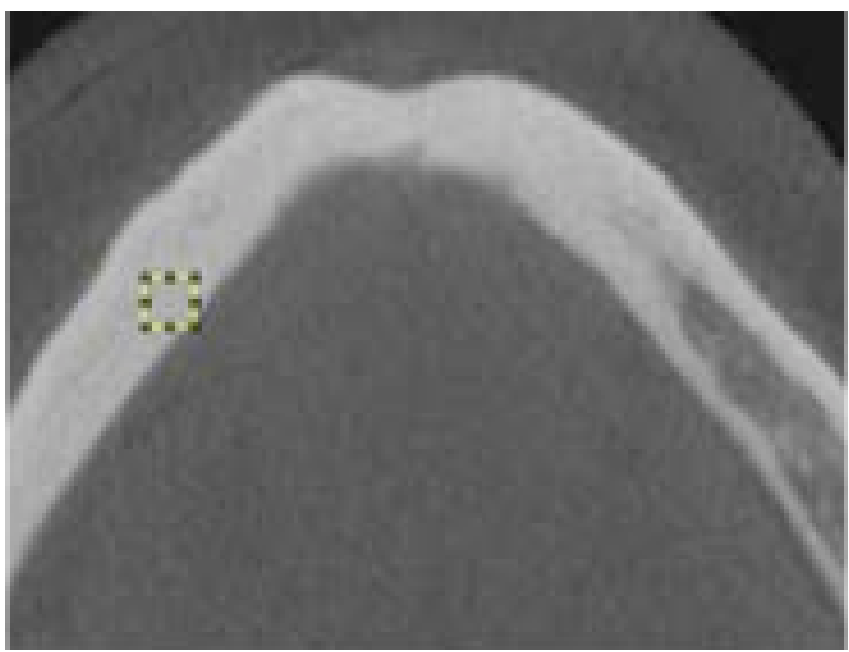

Figure 7. ROI of mental foramen in i-Cat. 


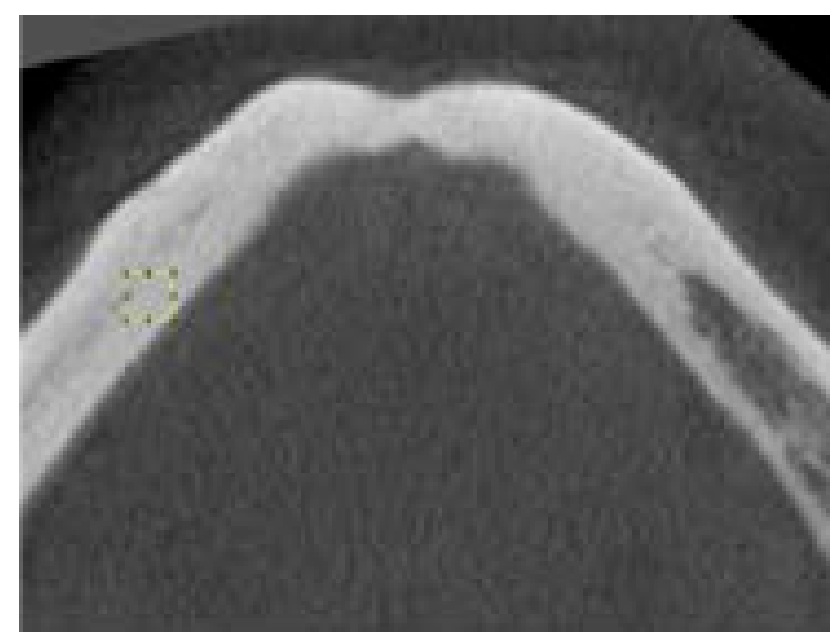

Figure 8. ROI of mental foramen in PreXion 3D.

With these coordinates set, ImageJ program then provided 36 and 57 ROI's of the mentum (with $40 \times$ 15 and $64 \times 24$ pixel resolution) and 43 and 70 ROI's of the mentual foramen (with $16 \times 16$ and $26 \times 26$ pixels resolution) from i-Cat and Prexion 3D scanners, respectively, all containing only bone structure for fractal analysis. Three comparisons between the measurements obtained from the fractal analysis were made between the two tomography scanners: the first, among the fractal analysis of the mentum region. The second, between the fractal analysis of the region of the mental foramen. And the third, between the fractal analysis of the two regions together.

Then, all selected ROI's were duplicated and converted from 16 to 8 bits. Then the images were once again duplicated and darkened by a Gaussian filter with a 35-pixel diameter, step in which the whole structure was removed in fine and medium scale and only large variations in density were maintained. In the next step, this image was subtracted from the original image. In the resulting image, 128 grayscale was assigned. The next step was to transform the resulting image into a binary image, in order to perform the erosion step. In order to dilate the image, each pixel was replaced with the maximum value of the neighboring pixels. In the inversion, the image of the previous result was inverted, and the image changed from white to black and vice versa, making it easier to count. Then, skeletonization was performed. In this process, the pixels of the edges of the images were removed until the image was reduced to a single broad skeleton the size of a pixel. The analysis was carried out in the skeletized images, generating, finally, the values of the fractal dimension. In order to confirm the results obtained, the skeletonized image was superimposed on the initial image, ensuring that the skeletonization corresponded to the original image.

\subsubsection{Statistical Analysis}

A single evaluator, experienced in CBCT images, performed the selection of the tomographic images and the fractal analysis and the intraclass correlation coefficient determined the intra-examiner reliability. This was calculated by determining the fractal analysis of 15 randomly selected images, measured twice, with a 30 day interval between measurements. To verify the normality of the data, Shapiro-Wilk test was applied. The Mann-Whitney U, Levene and T tests were applied in order to compare the fractal analysis obtained by the two CBCT scanners.

Statistical analysis was performed with significance level of 5\% and processed in SPSS Statistics 23.0 (IBM Corporation, Armonk, USA).

\section{RESULTS}

The intraclass correlation coefficient result was 0.988 , indicating a high similarity between the measurements. 
Three comparisons between the measurements obtained from the fractal analysis were made between the two scanners. It was found that the mean of the samples in the three comparisons was higher for PreXion 3D scanner (Table 1). A normality test was performed for each comparison group. For the mentum samples, only those from the i-Cat scanner did not match the normal distribution. For the mental foramen samples, only those from the PreXion 3D scanner did not match the normal distribution. In the comparison of the samples together, the measurements of fractal analysis matched the normal distribution (Table 2).

Because in mentum and mental foramen comparisons at least one scanner sample did not match the normal distribution, the Mann-Whitney $\mathrm{U}$ test was applied, a nonparametric alternative to the t-test to compare the means of two groups. The $\mathrm{p}$ value of the test was 0.000 , for both comparisons, indicating that there was a significant difference between the fractal dimensions of the images obtained with i-Cat and PreXion 3D scanners, at a 5\% confidence level.

The t-test was used in the third comparison, which included all mentum and mental foramen samples, as both devices offered samples that, when compared all together, matched the normal distribution. The Levene test evaluates the hypothesis that the group variances are the same. As the significance associated with the test was $\mathrm{p}=0.000$, we conclude that the variances are different. Observing the significance of t-test we noticed that, being lower than 0.05 , there is a difference between the fractal dimensions (Table 3).

Table 1. Descriptive statistics of fractal analysis.

\begin{tabular}{ccccccc}
\hline \multirow{2}{*}{$\begin{array}{c}\text { Descriptive } \\
\text { Statistics }\end{array}$} & \multicolumn{2}{c}{ Mentum } & \multicolumn{2}{c}{ Mental Forame } & Mentum and Mental Foramen \\
\cline { 2 - 7 } & i-Cat & PreXion 3D & i-Cat & PreXion 3D & i-Cat & PreXion 3D \\
\hline Images & 36 & 57 & 43 & 70 & 79 & 127 \\
Mean & 1.029 & $\mathbf{1 . 2 5 6}$ & 0.819 & 1.112 & 0.917 & 1.175 \\
$\begin{array}{c}\text { Standard } \\
\text { Deviation }\end{array}$ & 0.116 & $\mathbf{0 . 0 8 8}$ & 0.128 & $\mathbf{0 . 0 9 4}$ & 0.161 & $\mathbf{0 . 1 1 6}$ \\
Minimum & 0.824 & 1.044 & 0.538 & 0.866 & 0.538 & 0.866 \\
Maximum & 1.192 & 1.398 & 1.074 & 1.274 & 1.192 & 1.398 \\
\hline
\end{tabular}

Table 2. Normality test (Shapiro-Wilk).

\begin{tabular}{ccccccc}
\hline \multirow{2}{*}{ Shapiro-Wilk } & \multicolumn{2}{c}{ Mentum } & \multicolumn{2}{c}{ Mental Forame } & Mentum and Mental Foramen \\
\cline { 2 - 7 } & i-Cat & PreXion 3D & i-Cat & PreXion 3D & i-Cat & PreXion 3D \\
\hline Statistics & 0.925 & 0.962 & 0.981 & 0.941 & 0.974 & 0.984 \\
df & 36 & 56 & 41 & 0,69 & 77 & 125 \\
Sig. & $\mathbf{0 . 0 1 8}$ & 0.078 & 0.728 & $\mathbf{0 . 0 0 3}$ & 0.118 & 0.143 \\
\hline
\end{tabular}

Table 3. Independent sample test.

\begin{tabular}{cccccc}
\hline & & \multicolumn{2}{c}{ Levene Test } & \multicolumn{2}{c}{$t$ Test } \\
\cline { 2 - 6 } & Variance & $\mathrm{Z}$ & $\mathrm{Sig}$ & $\mathrm{t}$ & Sig. \\
\hline \multirow{2}{*}{ Sample measurements } & assumed equal & \multirow{2}{*}{14.899} & $\mathbf{0 . 0 0 0}$ & -13.190 & 0.000 \\
& not assumed & & & -12.213 & $\mathbf{0 . 0 0 0}$ \\
\hline
\end{tabular}




\section{DISCUSSION}

Recently, there has been a tendency to use conical beam computed tomography in dental exams [16]. Because of the distortion of CBCT measurement from dental CT values, there has been debate on whether CBCT measurement can be used to infer bone mineral densities $[9,17]$. Some relevant features of the bone structure, such as those based on fractal analysis, may still provide discriminative information to separate different trabecular patterns [18]. In the field of oral and maxillofacial radiology, the fractal analysis has been used to evaluate bone density $[9,10,19]$. According to Southard et al., in 1996 [20], there is a positive relation between the fractal dimension and the alveolar bone density. As the bone density increases, so does the fractal dimension. Using the fractal dimension of children's mandible images, Wojtowicz et al., in 2001 [21], showed the increasing complexity of trabecular patterns as the bone grew, and Nair et al., in 2001 [22], and Heo et al., in 2002 [23], reported that the fractal dimension increased during the bone recovery process.

There are several parameters that may influence the quality of a CT scan, such as FOV size, type of detector and reconstructed voxel size, and the unit itself [14]. These parameters vary between CBTC units and with different imaging protocols of the same unit [15]. The higher number of images selected from PreXion 3D scanner compared to i-Cat for the same bone segment is due to the fact that PreXion 3D presents a smaller voxel. The Image J program identifies and maintains the original voxel of the device used when creating new slices.

These parameters may lead to differences in image resolution and may be varied according to the diagnostic task, but protocols for specific diagnostic tasks in dentistry have not yet been established. Small-volume CBCT scanners (PreXion 3D) are known to generate higher resolution images compared to large-volume (i-Cat) CBCT scans (LEE, 2008). When performing the small volume CBCT, Wang et al., in 2011 [24], found higher levels of precision (91.9\%). In addition, large volume CBCT exposes patients to a higher dose of radiation compared to small volume [15] because of the greater area of incidence of x-rays. The selected images from i-Cat scanner presented $40 \times 15$ pixels and $16 \times 16$ pixels while those from PreXion 3D scanner presented $64 \times 24$ pixels and $26 \times 26$ pixels in the mentum region and the mental foramen respectively.

Van Dessel et al., in 2013 [25], compared the parameters of the bone structure at 90 and $70 \mathrm{kV}$, without setting the radiation dose (dose index: $3.4-8.1 \mathrm{mGy}$ ) and concluded that the $\mathrm{kV}$ effect was not as pronounced as the mA. Pawels et al., in 2015 [26], compared the fractal dimension and other parameters of the bone structure using three combinations of tube voltage $(\mathrm{kV})$ and tube exposure time $(\mathrm{mA})$. They concluded that most bone structure parameters are not affected by $\mathrm{kV}$ if the radiation dose is constant. However, the trabecular structure parameters, including the fractal dimension, were strongly affected by voxel size: they gradually decreased in larger voxel sizes. This is in agreement with the spatial resolution decreasing in larger voxel sizes [27] leading to a loss of detail in the trabecular bone, which can be seen as a "fusion" of adjacent trabeculae.

Baksi and Filder, in 2012 [28], when evaluating the effect of exposure time and image resolution on fractal dimension of periapical bone on images obtained using a storage phosphor plate system, found that images obtained with super-resolution scanning gave significantly higher fractal dimensions than high-resolution images for all exposures. The fractal dimension values decreased as the exposure time increased for both resolutions. Higher fractal dimension was found for super-resolution images and lower exposure time.

Fractal analysis of CBCT images is generally used in researches that use the same model of computerized tomography scans machines, only varying the parameters of the image taking. Fractal analysis is eventually used in conjunction with other analysis, such as: CT values, histogram analysis, dual-energy X-ray absorptiometry (DXA method) and bone mineral density (BMD) measured by DXA [29].

The differences in fractal dimension values reflect the bone density differences between individuals, and it may be impracticable to directly compare these values between individuals. This is supported by the fact that different studies on the fractal dimension of the normal maxillary bone produced different nor- 
mal values [19].

The methods of determining fractal dimension are extremely diverse, for example simplified spatial methods (such as the caliper method, tile counting method, and pixel dilation method), general spatial methods (such as the box counting method, intensity variance method, Hurst method, variation method, and blanket method), and spectral methods (such as spatial and spectral methods and the power spectrum method) [30]. Geraets and van der Stelt, in 2000 [30], found that the fractal dimensions reported in studies using fractal analysis for bone diseases differ according to the methods used. Then, because the fractal dimensions may differ according to the calculation methods used, it is necessary to unify the methods used for clinical applications.

In this study, images with higher resolution and higher averages of the fractal dimensions were obtained from PreXion 3D compared to those from i-Cat. Thus, the results of this study do not support the comparison of fractal dimensions between different CBCT scanners.

\section{CONCLUSION}

The result of this study allows us to conclude that all the tests comparing the averages of the fractal dimension of the images obtained with i-CAT and PreXion 3D showed statistically significant differences and that larger averages were observed in the fractal analysis from PreXion 3D.

\section{CONFLICTS OF INTEREST}

The authors declare no conflicts of interest regarding the publication of this paper.

\section{REFERENCES}

1. Palacios, E., Valvassori, G.E., Shannon, M. and Reed, C.F. (1990) Magnetic Resonance of the Temporomandibular Joint: Clinical Considerations, Radiography, Management. Thieme Medical Publishers, New York.

2. Silva, M.A., Wolf, U., Heinicke, F., Bumann, A., Visser, H. and Hirsch, E. (2008) Cone-Beam Computed Tomography for Routine Orthodontic Treatment Planning: A Radiation Dose Evaluation. American Journal of Orthodontics and Dentofacial Orthopedics, 133, 640.e1-640.e5.

https://www.ajodo.org/article/S0889-5406(08)00060-7/fulltext

3. Guerrero, M.E., Jacobs, R., Loubele, M., Schutyser, F., Suetens, P. and van Steenberghe, D. (2006) State-of-the-Art on Cone Beam CT Imaging for Preoperative Planning of Implant Placement. Clinical Oral Investigations, 10, 1-7. https://doi.org/10.1007/s00784-005-0031-2

4. Loubele, M., Maes, F., Schutyser, F., Marchal, G., Jacobs, R. and Suetens, P. (2006) Assessment of Bone Segmentation Quality of Cone-Beam CT versus Multislice Spiral CT: A Pilot Study. Oral Surgery, Oral Medicine, Oral Pathology, Oral Radiology and Endodontics, 102, 225-234. https://doi.org/10.1016/j.tripleo.2005.10.039

5. Kahl, B., Fischbach, R. and Gerlach, K.L. (1995) Temporomandibular Joint Morphology in Children after Treatment of Condylar Fractures with Functional Appliance Therapy: A Follow-Up Study Using Spiral Computed Tomography. Dentomaxillofacial Radiology, 24, 37-45. https://doi.org/10.1259/dmfr.24.1.8593906

6. Sato, H., Kawamura, A., Yamaguchi, M. and Kasai, K. (2005) Relationship between Masticatory Function and Internal Structure of the Mandible Based on Computed Tomography Findings. American Journal of Orthodontics and Dentofacial Orthopedics, 128, 766-773. https://doi.org/10.1016/j.ajodo.2005.05.046

7. Turkyilmaz, I., Tözüm, T.F. and Tumer, C. (2007) Bone Density Assessments of Oral Implant Sites Using Computerized Tomography. Journal of Oral Rehabilitation, 34, 267-272.

https://doi.org/10.1111/j.1365-2842.2006.01689.x

8. Hsu, J.T., Chang, H.W., Huang, H.L., Yu, J.H., Li, Y.F. and Tu, M.G. (2010) Bone Density Changes around Teeth during Orthodontic Treatment. Clinical Oral Investigations, 15, 511-519. 
https://doi.org/10.1007/s00784-010-0410-1

9. Hua, Y., Nackaerts, O., Duyck, J., Maes, F. and Jacobs, R. (2009) Bone Quality Assessment Based on Cone Beam Computed Tomography Imaging. Clinical Oral Implants Research, 20, 767-771.

https://doi.org/10.1111/j.1600-0501.2008.01677.x

10. Torres, S.R., Chen, C.S.K., Leroux, B.G., Lee, P.P., Hollender, L.G. and Schubert, M.M. (2011) Fractal Dimension Evaluation of Cone Beam Computed Tomography in Patients with Bisphosphonate-Associated Osteonecrosis. Dentomaxillofacial Radiology, 40, 501-505. https://doi.org/10.1259/dmfr/14636637

11. Campos, M.J., de Souza, T.S., Mota Júnior, S.L., Fraga, M.R. and Vitral, R.W. (2014) Bone Mineral Density in Cone Beam Computed Tomography: Only a Few Shades of Gray. World Journal of Radiology, 6, 607-612. https://doi.org/10.4329/wjr.v6.i8.607

12. Assis, T.A., Vivas Miranda, J.G., Mota, F.B., Andrade, R.F.S. and Castilho, C.M.C. (2008) Geometria Fractal: Propriedades e características de fractais ideais. Revista Brasileira de Ensino de Física, 30, 2304.1-2304.10.

13. Sánchez, I. and Uzcátegui, G. (2011) Fractals in Dentistry. Journal of Dentistry, 39, 273-292. https://doi.org/10.1016/j.jdent.2011.01.010

14. Kamburoglu, K., Murat. S., Kolsuz, E., Kurt, H., Yuksel, S. and Paksoy, C. (2011) Comparative Assessment of Subjective Image Quality of Cross-Sectional Cone-Beam Computed Tomography Scans. Journal of Oral Science, 53, 501-508. https://doi.org/10.2334/josnusd.53.501

15. Librizzi, Z.T., Tadinada, A.S., Valiyaparambil, J.V., Lurie, A.G. and Mallya, S.M. (2011) Cone-Beam Computed Tomography to Detect Erosions of the Temporomandibular Joint: Effect of Field of View and Voxel Size on Diagnostic Efficacy and Effective Dose. American Journal of Orthodontics and Dentofacial Orthopedics, 140, e25-e30. https://www.ajodo.org/article/S0889-5406(11)00326-X/fulltext

16. Alamri, H.M., Sadrameli, M., Alshalhoob, M.A., Sadrameli, M. and Alshehri, M.A. (2012) Applications of CBCT in Dental Practice: A Review of the Literature. General Dentistry, 60, 390-400.

17. Katsumata, A., Hirukawa, A., Noujeim, M., Okumura, S., Naitoh, M., Fujishita, M., Ariji, E. and Langlais, R.P. (2006) Image Artifact in Dental Cone-Beam CT. Oral Surgery, Oral Medicine, Oral Pathology, Oral Radiology and Endoddontology, 101, 652-657. https://doi.org/10.1016/j.tripleo.2005.07.027

18. Ling, H., Yang, X., Li, P., Megalooikonomou, V., Xu, Y. and Yang, J. (2014) Cross Gender-Age Trabecular Texture Analysis in Cone Beam CT. Dentomaxillofacial Radiology, 43, Article ID: 20130324.

https://doi.org/10.1259/dmfr.20130324

19. Southard, T.E., Southard, K.A. and Lee, A. (2001) Alveolar Process Fractal Dimension and Postcranial Bone Density. Oral Surgery, Oral Medicine, Oral Pathology, Oral Radiology and Endodontics, 91, 486-491. https://doi.org/10.1067/moe.2001.112598

20. Southard, T.E., Southard, K.A., Jakobsen, J.R., Hillis, S.L. and Najim, C.A. (1996) Fractal Dimension in Radiographic Analysis of Alveolar Process Bone. Oral Surgery, Oral Medicine, Oral Pathology, Oral Radiology and Endodontics, 82, 569-576. https://doi.org/10.1016/S1079-2104(96)80205-8

21. Wojtowicz, A., Chaberek, S., Pirino, A., Montella, A., Bandiera, P., Kinsner, A. and Ostrowski, K. (2001) The Trabecular Structure of Developing Human Mandible. Orthodontics and Craniofacial Research, 4, 161-171. https://doi.org/10.1034/j.1600-0544.2001.040306.x

22. Nair, M.K., Seyedain, A., Webber, R.L., Nair, U.P., Piesco, N.P., Agarwal, S., Mooney, M.P. and Gröndahl, H.G. (2001) Fractal Analyses of Osseous Healing Using Tuned Aperture Computed Tomography Images. European Radiology, 11, 1510-1515. https://doi.org/10.1007/s003300000773

23. Heo, M.S., Park, K.S., Lee, S.S., Choi, S.C., Koak, J.Y., Heo, S.J., Han, C.H. and Kim, J.D. (2002) Fractal Analysis of Mandibular Bony Healing after Orthognathic Surgery. Oral Surgery, Oral Medicine, Oral Pathology, Oral Radiololgy, and Endodontics, 94, 763-767. https://doi.org/10.1067/moe.2002.128972 
24. Wang, P., Yan, X.B., Lui, D.G., Zhang, W.L., Zhang, Y. and Ma, X.C. (2011) Detection of Dental Root Fractures by Using Cone-Beam Computed Tomography. Dentomaxillofacial Radiology, 40, 290-298. https://doi.org/10.1259/dmfr/84907460

25. Van Dessel, J., Huang, Y., Depypere, M., Rubira-Bullen, I., Maes, F. and Jacobs, R. (2013) A Comparative Evaluation of Cone Beam CT and Micro-CT on Trabecular Bone Structures in the Human Mandible. Dentomaxillofacial Radiology, 42, Article ID: 20130145. https://doi.org/10.1259/dmfr.20130145

26. Pauwels, R., Araki, K., Siewerdsen, J.H. and Thongvigitmanee, S.S. (2015) Technical Aspects of Dental CBCT: State of the Art. Dentomaxillofacial Radiology, 44, Article ID: 20140224. https://doi.org/10.1259/dmfr.20140224

27. Pauwels, R., Faruangsaeng, T., Charoenkarn, T., Ngonphloy, N. and Panmekiate, S. (2015) Effect of Exposure Parameters and Voxel Size on Bone Structure Analysis in CBCT. Dentomaxillofacial Radiology, 44, Article ID: 20150078.

28. Baksi, B.G. and Fidler, A. (2012) Image Resolution and Exposure Time of Digital Radiographs Affects Fractal Dimension of Periapical Bone. Clinical Oral Investigations, 16, 1507-1510.

https://doi.org/10.1007/s00784-011-0639-3

29. Mostafa, R.A., Arnout, E.A. and El-Fotouh, M.M. (2016) Feasibility of Cone Beam Computed Tomography Radiomorphometric Analysis and Fractal Dimension in Assessment of Postmenopausal Osteoporosis in Correlation with Dual X-Ray Absorptiometry. Dentomaxillofacial Radiology, 45, Article ID: 20160212.

30. Geraets, W.G. and van der Stelt, P.F. (2000) Fractal Properties of Bone. Dentomaxillofacial Radiology, 29, 144-153. https://doi.org/10.1038/sj.dmfr.4600524 